\title{
(2) OPEN ACCESS \\ Gene therapy for neovascular age-related macular degeneration: rationale, clinical trials and future directions
}

\author{
Thales Antonio Cabral de Guimaraes (D) ,'2 Michalis Georgiou (D) 1,2 \\ James W B Bainbridge, ${ }^{1,2}$ Michel Michaelides ${ }^{1,2}$
}

\section{'UCL Institute of Ophthalmology, University College London, London, UK ${ }^{2}$ Moorfields Eye Hospital, London, UK}

\section{Correspondence to}

Michel Michaelides, Moorfields Eye Hospital, London EC1V 9EL, UK;

michel.michaelides@ucl.ac.uk

Received 27 February 2020 Revised 18 March 2020 Accepted 20 March 2020 Published Online First 8 April 2020

\section{Check for updates}

(c) Author(s) (or their employer(s)) 2021. Re-use permitted under CC BY. Published by BMJ.

To cite: Guimaraes TAC, Georgiou M, Bainbridge JWB, et al. Br I Ophthalmol 2021:105:151-157.

\begin{abstract}
Age-related macular degeneration (AMD) is one of the leading causes of irreversible blindness in the developed world. Antivascular endothelial growth factor therapy has transformed the management and outcome of neovascular AMD (nAMD), although the need for repeated intravitreal injections - even lifelong — and the related complications, high drug costs, frequent clinic visits and repeated imaging have resulted in an enormous burden both to healthcare systems and patients. The application of gene therapy approaches for sustained delivery of a range of antiangiogenic proteins has the promise of helping to address these aforementioned challenges. A number of early phase clinical trials of gene therapy in nAMD have provided encouraging results, with many more ongoing or anticipated. There remain significant areas of controversy, including regarding the optimal treatment targets, routes of administration and potential safety concerns. In this review we aim to provide an update of the current status of gene therapy for nAMD and briefly discuss future prospects.
\end{abstract}

\section{INTRODUCTION}

Age-related macular degeneration (AMD) is a progressive disease that leads to severe impairment of central vision. It is a leading cause of irreversible blindness in the elderly population. ${ }^{1-3}$ A large systematic review and meta-analysis predicted the number of patients affected by the condition worldwide to be 288 million by $2040 .{ }^{4}$ In Europe alone, late AMD is expected to affect 77 million people by $2050 .^{5}$

AMD can be categorised into two main groups: dry or non-neovascular AMD, and wet or neovascular AMD. Neovascular AMD (nAMD) presents with the growth of abnormal vessels, most often emanating from the choroidal circulation, but can originate from the retinal vasculature, causing acute vision loss through leakage, and if chronic through fibrosis and atrophy. ${ }^{6}$ While a complex array of proangiogenic and antiangiogenic factors are involved in vascular homeostasis, it appears that nAMD is primarily driven by a perturbation of vascular endothelial growth factor (VEGF), demonstrated by the dramatic improvements observed in patients with nAMD following the development of anti-VEGF agents. This class of drug is now the gold standard treatment for nAMD. ${ }^{7}$ These intravitreal drugs inhibit neovascular proliferation by directly binding to VEGF or to VEGF receptors (VEGFR). However, the high drug costs and the frequent need for intravitreal injections have resulted in a significant (arguably unsustainable) burden, economically, logistically and socially. ${ }^{8}$ Long-term follow-up studies have shown that visual acuity (VA) gains during the first years of treatment were not maintained and that there was a progressive decline of VA gains achieved with repeated monthly treatment. ${ }^{9-11}$ Moreover, a recent meta-analysis suggested that treatment intensity correlated directly with final visual outcomes (with real-world experience often being of 'under-treatment' and thereby inferior outcomes compared with those achieved in pivotal clinical trials), further highlighting the need for a more sustainable release approach. ${ }^{12}$

Gene therapy offers the very real possibility of such a sustained delivery-in a 'one and done' manner. The gene therapy for RPE65-associated retinal dystrophy approved by the Food and Drug Administration and the European Medicines Agency has helped pave the way for a new era of retinal gene therapy products. ${ }^{13-15}$ Gene therapy for nAMD is on the verge of transforming management and outcomes, as intravitreal anti-VEGF agents did a decade ago. This review aims to provide an update on targets, trials and future directions.

\section{MOLECULAR BASIS OF ANGIOGENESIS IN NAMD}

Angiogenesis is a complex and critical physiological process which is tightly regulated by proangiogenic and antiangiogenic factors. ${ }^{16}$ In ocular disease, in the presence of hypoxia, retinal neurons such as the retinal ganglion cells release growth factors which upregulate the secretion of VEGF by Muller cells and retinal microglia. ${ }^{17}$ Although several factors are involved in this physiological process, including placental growth factor (PIGF), platelet-derived growth factor (PDGF- $\beta$ ), angiopoietin-1 (Ang1) and angiopoietin-2 (Ang2), VEGF plays the major role, primarily regulating vascular permeability and neovascularisation. ${ }^{16-22}$ It has multiple isoforms due to alternative splicing, although $\mathrm{VEGF}_{165}$ is the predominant isoform and most active in vasculogenesis. $^{23-25}$

Among VEGF's signalling receptors, the endothelial and neuron-expressed VEGFR1 (FLT1) and VEGFR2 (FLK1/KDR) are responsible for regulation of cell proliferation, migration and vascular permeability in ocular disease, whereas VEGFR3 


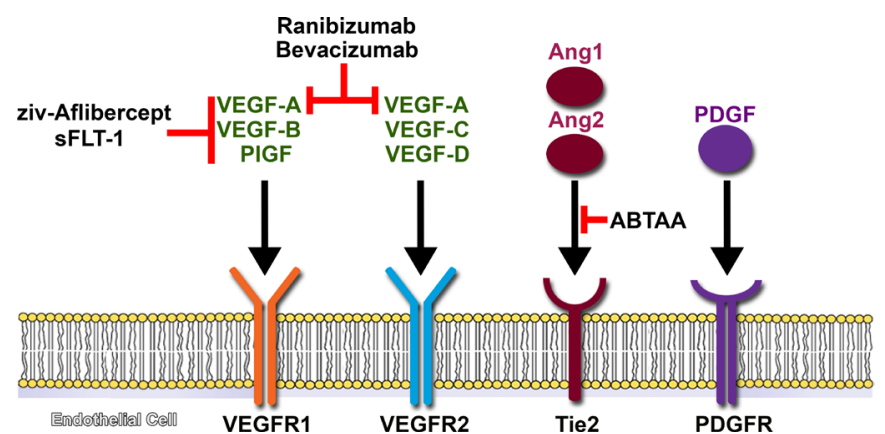

Figure 1 Promising targets for antiangiogenesis in $\mathrm{nAMD}$. The main goal of gene therapy in this condition is to continuously express antiangiogenic factors and lead to a more sustainable treatment. Some have already been extensively studied in the past for intravitreal treatment of nAMD, like bevacizumab and ranibizumab, while others like Angpt2-binding and Tie2-activating antibodies (ABTAA) are currently being investigated and might be useful for combination therapy. The platelet-derived growth factor (PDGF) pathway also represents another potential target as it mediates the recruitment and survival capabilities of pericytes and might be implicated in the development of subretinal fibrosis. Ang1, angiopoietin-1; Ang2, angiopoietin-2; nAMD, neovascular age-related macular degeneration; PIGF, placental growth factor; VEGF, vascular endothelial growth factor.

is mainly expressed in lymphatic endothelial cells and is mostly responsible for lymphangiogenesis. ${ }^{20}{ }^{26}$ VEGF-A binds with high affinity to both VEGFR1 and VEGFR2, while VEGF-B and PIGF bind exclusively to VEGFR1 (figure 1). ${ }^{27-29}$ Therefore, the vast majority of gene therapy trials for nAMD target VEGF, either directly or indirectly via its receptors, although other factors are also being investigated as possible targets and will be reviewed in the following sections.

\section{VIRAL VECTORS AND THE EYE}

The eye is arguably the ideal target for gene therapy: it has relative immune privilege, since it is separated from the rest of the body by the presence of the inner blood-retinal barrier; it is highly compartmentalised, which allows easy access to different and specific ocular tissues; the transparency of the ocular media allows the direct visualisation of the retina; and visual function and retinal architecture can be monitored readily using non-invasive methods. ${ }^{30}$ A prerequisite for gene therapy to be successful is a vector that leads to sustained levels of gene expression, low toxicity and immunogenicity. Various non-viral and viral vector systems have been used in different settings, but the most suitable for retinal gene supplementation is the recombinant adeno-associated viral vector (AAV). ${ }^{31} 32$

A major reason for the success of AAV is its small, singlestranded DNA genome of about 4.6 kilobases $(\mathrm{kb})$ and capsid organisation, which facilitate genetic modification, thus enabling customisation of its properties. ${ }^{33}$ However, given the fact that approximately $6 \%$ of all human proteins have a coding sequence bigger than $4 \mathrm{~kb}$, this small packaging capability is also a limitation of the first-generation AAV2. ${ }^{34}$ Several strategies have been investigated to bypass this cargo capacity, including directed evolution and the generation of dual AAV vectors. The latter aims to reconstitute a larger gene by either trans-splicing, homologous overlapping sequences or a combination of both. ${ }^{36}$ Other AAV serotypes have also been identified, but the most extensively studied in ocular gene therapies are AAV2, AAV5 and AAV8. ${ }^{37-39}$
One of the principal considerations of gene therapy is the potential immune response to AAV capsid, which may have a damaging tissue effect, but might also mitigate therapeutic benefit. AAV vector administration in humans, unlike most animal models, results in antigen-specific T-cell activation. ${ }^{40}$ The risk is higher during the early postoperative period. A broad range of immunosuppressive agents (both steroids and steroidsparing agents), routes of administration (topical, periocular, intravitreal and oral) and duration (days to weeks to months) have been employed in ocular gene therapy trials, with the rationale that a relatively short course of immunosuppression in the perioperative period will ameliorate immune responses until the capsid antigens are cleared from the infected cells. ${ }^{40}$ As would be anticipated, studies have also demonstrated that there is a doseresponse relationship between humoral immunity to AAV capsid and dose of injected AAV particles. ${ }^{41}$ However, this relationship ultimately limits the maximum viral particle dose that can be delivered, and may in certain situations inherently limit therapeutic benefit, thereby additionally making optimal management (ideally prevention) of immune responses critical. The route of vector delivery, as discussed in the following section, also has a major direct impact on immunogenicity, with evidence to date indicating that subretinal delivery is associated with less inflammation than the intravitreal route. ${ }^{42}$

An additional consideration, in direct contrast to the majority of subjects with inherited retinal diseases (IRDs) who may be targeted with gene therapy, patients with nAMD will be significantly older. This may be advantageous in terms of the likely less rigorous immune responses anticipated in older people compared with children/young adults, but also potentially disadvantageous, as older patients may be more susceptible to possible deleterious effects of systemic immunosuppression and also have pre-existing medical conditions, making them ineligible to participate and/or more likely to have side effects.

\section{ROUTES OF VECTOR DELIVERY}

The route of administration is another major determinant of the efficacy of gene delivery. It allows the targeting of specific cell types by delivering the vector as close as possible to the desired tissue. ${ }^{43}$ Figure 2 offers a schematic view of the three principal modes of delivery: subretinal, intravitreal and suprachoroidal.

Although more complex and invasive than intravitreal injections, subretinal delivery is arguably preferable for diseases primarily affecting the retinal pigment epithelium (RPE) and/ or photoreceptors. Since most IRDs affect either of these two cell types or both, subretinal delivery is the most commonly used route of administration in gene therapy trials for most monogenic conditions. However, intravitreal delivery has been undertaken for (1) the two X linked retinoschisis (XLRS) gene therapy trials (NCT02317887 and NCT02416622), with one stopping due to lack of efficacy and inflammation concerns (NCT02416622); and also (2) delivery of antisense oligonucleotide therapies for CEP290 and USH2A variants (NCT03140969 and NCT03780257, respectively), although these do not require a viral vector. While subretinal delivery with its associated transient retinal detachment is likely to be inherently associated with greater tissue damage/disturbance compared with intravitreal, the available trial data suggest that it is well tolerated, safe and may provide effective treatment. ${ }^{13} 1544$ The site of subretinal injection is also an important consideration. A retinotomy being near the (often initial retinotomy being superior) temporal vascular arcades is desirable as it allows the bleb to spread more gently towards the fovea and create a shallow elevation of the foveal 
A

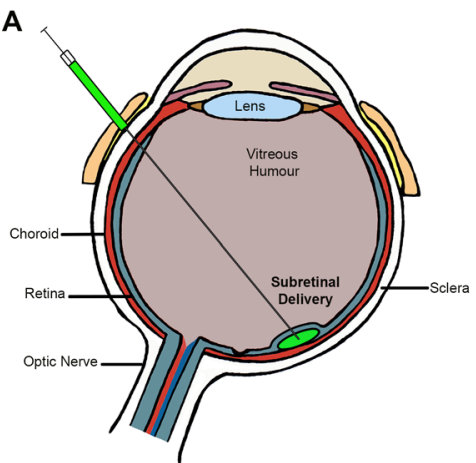

B

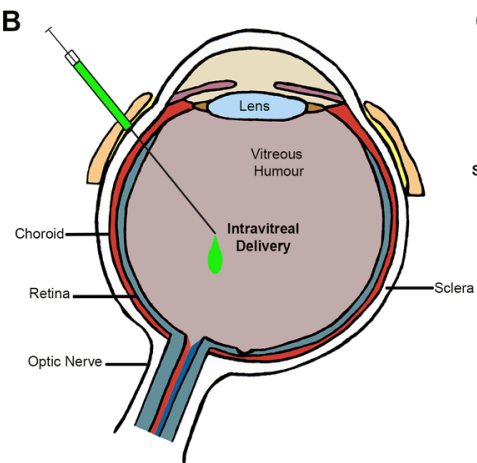

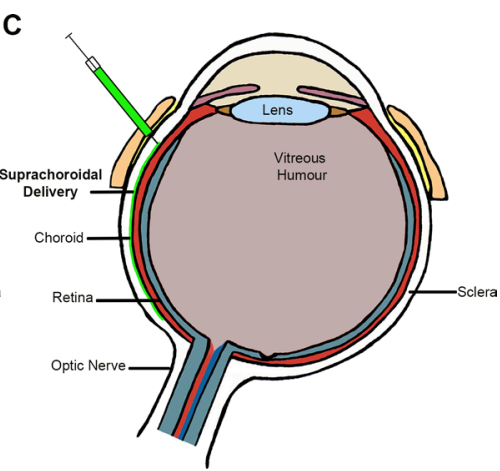

Figure 2 Delivery routes for gene therapy. Schematic figure of the eye demonstrating the possible routes of delivery to introduce viral vectors into the eye.

region, thereby also minimising foveal stretch and reducing the deleterious effects of a localised central foveal detachment. ${ }^{45-47}$

Intravitreal injection of anti-VEGF agents has become the mainstay of treatment for nAMD, diabetic eye disease and other retinovascular diseases. Although much less invasive than subretinal injections and readily delivered by non-specialist surgeons, the majority of currently available AAVs are unable to reach the outer retina, including the RPE and choroid, mainly due to the inner limiting membrane (ILM) acting as a physical barrier, thereby primarily limiting transduction to the inner retinal layers. However, modified AAVs (especially serotypes 2, 8 and 9 , with or without modified capsid proteins) are proposed to be more effective in penetrating the ILM and able to result in broader transduction. ${ }^{48-52}$ Primate data using intravitreal delivery of AAV2 have shown an annulus of high transduction in the perifoveal zone ('ring-of-fire'). ${ }^{50}$ This result differs from the more uniform transduction reported after intravitreal injection in other species, including rodents. ${ }^{5354}$ Additionally and equally important are immunological considerations, with intravitreal AAV administration in animal models shown to generate a humoral response against adenoviral capsid blocking further vector expression in the second eye, which did not happen with subretinal administration. ${ }^{42}$ Moreover, the aforementioned XLRS human clinical trial (NCT02416622) also demonstrated that the intravitreal route is more proinflammatory compared with subretinal delivery. In addition to being a significant safety concern, such immune response will likely mitigate any therapeutic effect.

Suprachoroidal delivery has also been explored and is arguably an attractive route for nAMD and other conditions affecting the choroid. Preclinical and animal data suggest that administration via suprachoroidal injection results in more posterior and circumferential distribution of the agent. ${ }^{55}$ Widespread expression in the RPE and photoreceptor layers has been reported 2 weeks after suprachoroidal injection using a suprachoroidal space (SCS) microinjector of AAV8 expressing an anti-VEGF Fab (AAV8.GFP) in non-human primates, pigs and rats. ${ }^{56}$ In this preclinical study, delivery of RGX-314 into the SCS resulted in similar suppression of VEGF-induced vascular leakage and expression of anti-VEGF Fab as subretinal delivery. Previously, access to this virtual space, between the choroid and the sclera, has been investigated by different methods, including sclerotomy. In 2011, Peden et $a l^{57}$ described a novel technique in animal models, consisting of an ab externo approach using an illuminated microcatheter (originally designed for use in Schlemm's canaloplasty) to inject the vector into the SCS. ${ }^{5859}$ Subsequently, researchers used the iTrack microcatheter (iTRACK 400; iScience Interventional Corporation, Menlo Park, California, USA) to deliver a combination of bevacizumab and triamcinolone to the submacular SCS of 21 human subjects with nAMD. ${ }^{60}$ These patients were followed for a 6-month period. Complications were mild, with one eye experiencing a transient intraocular pressure elevation at 3 months postprocedure and two eyes had an apparent increase in pre-existing nuclear sclerotic cataract. ${ }^{60}$ The results of a phase II study to evaluate suprachoroidal injection of triamcinolone acetonide suspension in patients with macular oedema due to non-infectious uveitis (NCT02255032) were recently published with good tolerability and significantly improved VA. ${ }^{61}$

\section{GENE THERAPIES FOR NAMD}

Gene therapy holds the potential to continuously express therapeutic levels of angiostatic proteins. The completed and ongoing gene therapy trials for nAMD are summarised in table 1 and discussed in detail in the following sections.

\section{Pigment epithelium derived factor}

The intravitreal administration of a transgene vector of AAV5 carrying pigment epithelium derived factor (PEDF)

\begin{tabular}{|c|c|c|c|c|c|c|c|c|}
\hline Trial registration number & Expressed gene & Vector & Phase & Route of delivery & Status & Sponsor & Location & Patients (n) \\
\hline NCT00109499 & PEDF & AAV5 & 1 & Intravitreal & Completed & GenVec & USA & 28 \\
\hline NCT01494805 & sFLT01 & AAV2 & $\mid / I I$ & Subretinal & Completed & $\begin{array}{l}\text { Lions Eye Institute, } \\
\text { Adverum Biotechnologies }\end{array}$ & Australia & 40 \\
\hline NCT03748784 & Aflibercept & AAV2 & 1 & Intravitreal & Ongoing & Adverum Biotechnologies & USA & 30 \\
\hline NCT01024998 & sFLT01 & AAV2 & 1 & Intravitreal & Completed & Sanofi Genzyme & USA & 19 \\
\hline NCT03066258 & Anti-VEGF Fab & AAV8 & I/lla & Subretinal & Ongoing & Regenxbio & USA & 42 \\
\hline NCT01301443 & Endostatin and angiostatin & EIAV & 1 & Subretinal & Completed & Oxford BioMedica & USA & 21 \\
\hline NCT03585556 & sCD59 & AAV2 & I & Intravitreal & Ongoing & Hemera Biosciences & USA & 25 \\
\hline
\end{tabular}

$A A V$, adeno-associated viral vector; EIAV, equine infectious anaemia lentiviral vector; $\mathrm{AMMD}$, neovascular age-related macular degeneration. 
(AdGVPEDF.11D) in three murine models of choroidal neovascularization (CNV) significantly inhibited neovascularisation. ${ }^{62}$ This study led to one of the first gene therapy trials for nAMD (NCT00109499), a phase I, dose-escalation intravitreal delivery of AdPEDF.11. Although 25\% of the cohort showed mild signs of inflammation, such as keratic precipitates and flare in the anterior chamber, this was readily managed, and the authors deemed this gene therapy product to be safe and well tolerated. ${ }^{63}$ No conclusions regarding efficacy were made as the trial did not have randomised control groups. However, patients receiving $10^{8}$ particle units (PU) or more of AdPEDF.11 were significantly more likely to remain stable or have a reduction in the size of CNV lesions than patients who received less than $10^{8}$ $\mathrm{PU}$, suggesting a dose-related response.

\section{sFLT-1}

sFLT-1 is a highly potent endogenous VEGF-A inhibitor. ${ }^{64}$ The Lions Eye Institute, in collaboration with Adverum Biotechnologies, completed a phase I/II randomised controlled trial involving a single subretinal administration of rAAV.sFLT-1, also known as AVA-101 (NCT01494805). All patients $(\mathrm{n}=8)$ received additional ranibizumab injections at baseline, week 4 and as needed afterwards according to prespecified rescue criteria. AVA-101 was shown to be safe, thereby leading to an expansion phase, ${ }^{6566}$ with 32 patients randomised to either the $10^{11} \mathrm{vg}$ cohort or the control group. There were no serious adverse events and patients in the therapy cohort required a median of two ranibizumab injections compared with four for the control group. Although no significant improvement in best corrected VA was seen, safety and tolerability were demonstrated, supporting the feasibility of further ocular gene therapies as a potential long-term treatment option for nAMD.

Another phase I, dose-escalation study, sponsored by Sanofi Genzyme, evaluated the safety profile of a single intravitreal AAV2-sFLT-1 injection in patients with advanced nAMD (NCT01024998). It is similar to AVA-101, except that it is a fusion protein of the sFLT-1 domain 2 with the Fc domain of IgG1. Nineteen patients were divided into four dose cohorts and followed up for 52 weeks. Four subjects showed substantial and sustained macular fluid reduction through week 52, with reductions of central subfield thickness (CST) ranging from $128 \mu \mathrm{m}$ to $443 \mu \mathrm{m}$. Improvements in VA may have been limited due to pre-existing subretinal fibrosis/scarring and variability in baseline fluid. Overall the intravitreal injection was well tolerated at all doses, although one patient had transient intraocular inflammation at the highest dose $\left(2 \times 10^{10} \mathrm{vg}\right)$. Another two patients had retinal haemorrhage, one had multiple retinal tears 1 month after injection, and there was a death in the study, with none of these believed to be associated with the procedure or the gene therapy product. ${ }^{67}$ Additionally, five out of ten subjects who received the high dose had no detectable anti-AAV2 serum antibodies. One of these patients showed no detectable sFLT-1 in the aqueous humour despite undetectable anti-AAV2 antibodies, indicating that anti-AAV2 titre and vector dose are not the only factors influencing transgene expression. ${ }^{67}$

\section{Aflibercept}

Preclinical evaluation of intravitreal ADVM-022 in non-human primates was well tolerated and effective at preventing clinically relevant laser-induced CNV lesions. ${ }^{68}$ Adverum Biotechnologies has started a phase I, dose-escalation trial (NCT03748784) to study the safety profile of intravitreal ADVM-022 (AAV2.7m8aflibercept), a novel recombinant AAV. Preliminary results of this human trial reveal that through week 34 , in all six patients of the first cohort, the gene therapy product was well tolerated with no serious adverse events noted. Nineteen adverse events related to inflammation were noted, with the vast majority considered mild and treated with topical steroid drops only. No retinitis, vasculitis or choroiditis was seen. In addition, the improvement in CST was sustained after 34 weeks and no rescue injections were needed. ${ }^{69} 70$ The last update was to a median follow-up of 44 weeks, no rescue injections were needed, and anatomical and vision improvements were sustained, although one patient had spontaneous pseudophakic retinal detachment, deemed to be unrelated to the Advanced Therapy Investigational Medicinal Product. ${ }^{71}$ The study is still ongoing and has an estimated completion date of February 2022.

\section{Ranibizumab-like}

RGX-314 is a recombinant AAV8 vector carrying a coding sequence for a soluble anti-VEGF protein related to ranibizumab. Regenxbio is conducting a phase I/II, dose-escalation study to evaluate the safety and tolerability of subretinal RGX-314 in subjects who have been previously treated with any anti-VEGF drug (NCT03066258). Forty-two patients were recruited and divided into five cohorts of $3 \times 10^{9} \mathrm{vg}, 1 \times 10^{10} \mathrm{vg}, 6 \times 10^{10} \mathrm{vg}, 1.6 \times 10^{11}$ and $2.5 \times 10^{11} \mathrm{vg}$. The trial is currently ongoing, with preliminary results being promising. In cohort $5,73 \%$ of subjects ( 8 of 11 ) remained free of needing any anti-VEGF injections for 6 months, associated with improvement in vision and retinal thickness. ${ }^{72}$ After 1.5 years of RGX-314 administration, $50 \%$ of cohort 3 ( 3 of 6 ) remain free of anti-VEGF drugs and have maintained the improvement in vision. Furthermore, RGX-314 was well tolerated at all doses and no inflammation beyond what is expected following routine vitrectomy was seen. ${ }^{72-74}$ Regenxbio is now planning to start suprachoroidal delivery trials for nAMD and diabetic retinopathy in $2020 .^{72}$

\section{Angiostatin and endostatin}

RetinoStat is an equine infectious anaemia lentiviral vector that expresses angiostatin and endostatin, both of which are inhibitors of angiogenesis. Animal models of rhesus macaques and rabbits have shown that subretinal administration was safe and capable of persistent expression. ${ }^{75}$ In a phase I, dose-escalation trial, 21 patients were enrolled into three cohorts. Although one patient developed a procedure-related macular hole, the subretinal injection of RetinoStat was safe and well tolerated, resulting in sustained transgene expression of VEGF-neutralising proteins (NCT01301443), established via analysis of aqueous samples. ${ }^{76}$ A concomitant long-term follow-up study is currently under way (NCT01678872).

\section{Complement cascade}

Hemera Biosciences recently started two phase I trials using intravitreal AAVCAGsCD59 for both nAMD and dry AMD (NCT03585556 and NCT03144999, respectively). This molecule targets the terminal step of complement activation that leads to the formation of the membrane attack complex. ${ }^{77}$ Interestingly, it has been shown that subretinal injection of AAV-CD59 attenuated the formation of laser-induced CNV by around $60 \%$ in mice, even when the site of delivery was distal to the laser-induced CNV site. ${ }^{78}$

\section{OTHER POSSIBLE TARGETS AND APPROACHES FOR GENE THERAPY}

Gene therapies for nAMD are largely focused on the use of vector systems to express antiangiogenic proteins that either directly or indirectly block the VEGF pathway as discussed earlier. Other 
possible targets for gene therapy are discussed in the following sections and presented in figure 1 .

\section{Platelet-derived growth factor}

PDGF mediates the recruitment and survival capabilities of pericytes. PDGF has been implicated in the pathogenesis of several fibrotic conditions and may also play a role in the development of subretinal fibrosis. ${ }^{79} 80$

\section{Tie- 2 tyrosine kinase receptor}

Tie-2 tyrosine kinase receptor is a potential target present in endothelial cells. The angiopoietin (Angpt)-Tie2 system functions as a key regulator of adult vascular homeostasis. ${ }^{81}$ In animal models, intravitreal administration of Ang1 was as effective as VEGF-trap in inhibiting CNV formation. Furthermore, Ang1 suppressed vascular leakage by increasing endothelial junctional proteins. ${ }^{82}$ More recently, an intravitreal dual functioning antibody, Angpt2-binding and Tie2-activating (figure 1), has been shown to suppress CNV formation and vascular leakage, and also improve hypoxia surrounding the CNV, thereby making it an attractive option for combination therapy with anti-VEGF drugs. ${ }^{83}$

\section{Gene silencing}

Gene silencing has been attempted via small RNA interference (siRNA), which has been shown to successfully suppress expression of VEGF in mice models. ${ }^{84}$ However, a phase III human trial administering the siRNA bevasiranib intravitreally (NCT00499590) was discontinued as recommended by the study's Independent Data Monitoring Committee due to the unlikelihood of reaching its primary objective, defined as the proportion of patients at week 60 in each group with a successful VA outcome.$^{85}$ Similarly, a phase II trial administering Sirna-027 (NCT00395057) failed to meet key efficacy endpoints, despite positive reports of the phase I/II study (NCT00363714). ${ }^{86}$

In a study by Askou et $a l^{87}$, AAV2/8-mediated subretinal delivery of a short hairpin RNA targeting VEGF has been found to be efficient and to reduce formation of $\mathrm{CNV}$ in preclinical murine models of wet AMD. An alternative to siRNAs is the use of microRNAs, a method that has been previously investigated by the same group of researchers. Intramuscular delivery of AAV containing polycistronic miRNA clusters efficiently silenced VEGF as early as 21 days after delivery in mice. ${ }^{88}$

\section{CONCLUSIONS AND FUTURE DIRECTIONS}

In summary, AMD is a complex disease with a multitude of pathways involved in its pathogenesis, with this heterogeneity posing therapeutic challenges. The approach to date has been to continuously express anticomplement and/or antiangiogenic proteins (especially targeting VEGF). There is no doubt that gene therapy holds promise to ameliorate the social and economic burdens associated with chronic anti-VEGF therapy by providing reliable and sustainable release of antiangiogenic drugs.

Several early phase trials have shown significant promise, with final results expected from the associated expansion phases in the near future. The competitive landscape revolves around multiple issues, including (1) the therapeutic target, (2) route of administration and (3) preventing/mitigating immune-mediated responses to viral vector administration. It is likely that antiVEGF-directed gene therapies will be the first to be approved, whereas the route of administration remains more uncertain, with intravitreal, subretinal and suprachoroidal all being actively investigated and all having relative merits and disadvantages.
With respect to inflammation, the nAMD patient population is significantly older than the IRD population receiving gene therapy, which may pose difficulties with tolerability to any systemic immunosuppressive drugs used in the preoperative and postoperative period, but alternatively older patients may be less likely to mount as robust an immune reaction than younger subjects.

Gene therapy allows the exciting possibility of expressing more than one active agent over time, with the capability of additional targets to VEGF, potentially resulting in greater efficacy, more sustained effect and being beneficial in patients who currently are poor responders to serial intravitreal anti-VEGFs in routine clinic use. Moreover, a greater understanding of the underlying drivers of inflammation in ocular gene therapy and its prevention/management is needed, as are developments in viral vectors that can be safely delivered intravitreally and have robust expression profiles. These two developments will arguably be game-changing, resulting in safe, readily delivered therapy to millions of patients worldwide with nAMD and potentially other common disorders including diabetic eye disease.

It is anticipated that the number of gene therapy trials will increase dramatically over the next 5 years, with cautious optimism of approved therapies within that timeframe.

Funding This work has been supported by grants from the National Institute for Health Research Biomedical Research Centre at Moorfields Eye Hospital NHS Foundation Trust and UCL Institute of Ophthalmology, Fight for Sight, Moorfields Eye Hospital Special Trustees, Moorfields Eye Charity, Retina UK, the Foundation Fighting Blindness (USA), and the Wellcome Trust (099173/Z/12/Z). The views expressed are those of the authors and not necessarily those of the NHS, the UCL, the NIHR or the Department of Health.

Competing interests MM, JWBB and MG consult for MeiraGTX.

\section{Patient consent for publication Not required.}

Provenance and peer review Not commissioned; externally peer reviewed.

Open access This is an open access article distributed in accordance with the Creative Commons Attribution 4.0 Unported (CC BY 4.0) license, which permits others to copy, redistribute, remix, transform and build upon this work for any purpose, provided the original work is properly cited, a link to the licence is given, and indication of whether changes were made. See: https://creativecommons.org/ licenses/by/4.0/.

\section{ORCID iDs}

Thales Antonio Cabral de Guimaraes http://orcid.org/0000-0002-7936-6851 Michalis Georgiou http://orcid.org/0000-0001-6397-8071

\section{REFERENCES}

1 Jin G, Ding X, Xiao W, et al. Prevalence of age-related macular degeneration in rural southern China: the Yangxi eye study. Br J Ophthalmol 2018;102:625-30.

2 Keel S, Xie J, Foreman J, et al. Prevalence of age-related macular degeneration in Australia: the Australian National eye health survey. JAMA Ophthalmol 2017; 135:1242-9.

3 Wilde C, Poostchi A, Mehta RL, et al. Prevalence of age-related macular degeneration in an elderly UK Caucasian population-The Bridlington eye assessment project: a cross-sectional study. Eye 2017;31:1042-50.

4 Wong WL, Su X, Li X, et al. Global prevalence of age-related macular degeneration and disease burden projection for 2020 and 2040: a systematic review and metaanalysis. Lancet Glob Health 2014;2:e106-16.

5 Li JQ, Welchowski T, Schmid M, et al. Prevalence and incidence of age-related macular degeneration in Europe: a systematic review and meta-analysis. Br J Ophthalmol 2020;104:1077-84.

6 Ferris FL, Wilkinson CP, Bird A, et al. Clinical classification of age-related macular degeneration. Ophthalmology 2013;120:844-51.

7 Lai K, Landa G. Current choice of treatments for neovascular AMD. Expert Rev Clin Pharmacol 2015;8:135-40.

8 Haller JA. Current anti-vascular endothelial growth factor dosing regimens: benefits and burden. Ophthalmology 2013;120:S3-7.

9 Krüger Falk M, Kemp H, Sørensen TL. Four-Year treatment results of neovascular age-related macular degeneration with ranibizumab and causes for discontinuation of treatment. Am J Ophthalmol 2013;155:89-95. 
10 Comparison of Age-related Macular Degeneration Treatments Trials (CATT) Research Group, Maguire MG, Martin DF, et al. Five-Year outcomes with anti-vascular endothelial growth factor treatment of neovascular age-related macular degeneration: the comparison of age-related macular degeneration treatments trials. Ophthalmology 2016; 123:1751-61.

11 Singer MA, Awh CC, Sadda S, et al. Horizon: an open-label extension trial of ranibizumab for choroidal neovascularization secondary to age-related macular degeneration. Ophthalmology 2012;119:1175-83.

12 Hussain RM, Hariprasad SM, Ciulla TA. Treatment Burden in Neovascular AMD:Visual Acuity Outcomes are Associated With Anti-VEGF Injection Frequency. Ophthalmic Surg Lasers Imaging Retina 2017;48:780-4.

13 Bainbridge JWB, Mehat MS, Sundaram V, et al. Long-Term effect of gene therapy on Leber's congenital amaurosis. N Eng/ J Med 2015;372:1887-97.

14 Testa F, Maguire AM, Rossi S, et al. Three-Year follow-up after unilateral subretinal delivery of adeno-associated virus in patients with Leber congenital amaurosis type 2 . Ophthalmology 2013;120:1283-91.

15 Weleber RG, Pennesi ME, Wilson DJ, et al. Results at 2 years after gene therapy for Rpe65-deficient Leber congenital amaurosis and severe Early-Childhood-Onset retinal dystrophy. Ophthalmology 2016;123:1606-20.

16 Rosen LS. Clinical experience with angiogenesis signaling inhibitors: focus on vascular endothelial growth factor (VEGF) blockers. Cancer Control 2002;9:36-44.

17 Apte RS, Chen DS, Ferrara N. Vegf in signaling and disease: beyond discovery and development. Cell 2019;176:1248-64.

18 Rakic J-M, Lambert V, Devy L, et al. Placental growth factor, a member of the VEGF family, contributes to the development of choroidal neovascularization. Invest Ophthalmol Vis Sci 2003;44:3186-93.

19 Ucuzian AA, Gassman AA, East AT, et al. Molecular mediators of angiogenesis. J Burn Care Res 2010:31:158-75.

20 Takahashi H, Shibuya M. The vascular endothelial growth factor (VEGF)/VEGF receptor system and its role under physiological and pathological conditions. Clin Sci 2005; 109:227-41.

21 Ferrara N. Vascular endothelial growth factor: basic science and clinical progress. Endocr Rev 2004;25:581-611.

22 Spilsbury K, Garrett KL, Shen WY, et al. Overexpression of vascular endothelial growth factor (VEGF) in the retinal pigment epithelium leads to the development of choroidal neovascularization. Am J Pathol 2000;157:135-44.

23 Harper SJ, Bates DO. Vegf-A splicing: the key to anti-angiogenic therapeutics? Nat Rev Cancer 2008;8:880-7.

24 Dehghanian F, Hojati Z, Kay M. New insights into VEGF-A alternative splicing: key regulatory switching in the pathological process. Avicenna J Med Biotechnol 2014:6:192-9.

25 Arcondéguy T, Lacazette E, Millevoi S, et al. Vegf-A mRNA processing, stability and translation: a paradigm for intricate regulation of gene expression at the posttranscriptional level. Nucleic Acids Res 2013;41:7997-8010.

26 Saharinen P, Tammela T, Karkkainen MJ, et al. Lymphatic vasculature: development, molecular regulation and role in tumor metastasis and inflammation. Trends Immunol 2004;25:387-95.

27 Terman $\mathrm{BI}$, Dougher-Vermazen M, Carrion ME, et al. Identification of the KDR tyrosine kinase as a receptor for vascular endothelial cell growth factor. Biochem Biophys Res Commun 1992:187:1579-86.

28 Maglione D, Guerriero V, Viglietto G, et al. Isolation of a human placenta cDNA coding for a protein related to the vascular permeability factor. Proc Natl Acad Sci U S A 1991;88:9267-71.

29 de Vries C, Escobedo JA, Ueno H, et al. The FMS-like tyrosine kinase, a receptor for vascular endothelial growth factor. Science 1992;255:989-91.

30 Kumaran N, Michaelides M, Smith AJ, et al. Retinal gene therapy. Br Med Bull 2018;126:13-25.

31 Rabinowitz JE, Rolling F, Li C, et al. Cross-packaging of a single adeno-associated virus (AAV) type 2 vector genome into multiple AAV serotypes enables transduction with broad specificity. J Virol 2002;76:791-801.

32 Day TP, Byrne LC, Schaffer DV, et al. Advances in AAV vector development for gene therapy in the retina. Adv Exp Med Biol 2014;801:687-93.

33 Grimm D, Büning H. Small but increasingly mighty: latest advances in AAV vector research, design, and evolution. Hum Gene Ther 2017;28:1075-86

34 Trapani I. Adeno-Associated viral vectors as a tool for large gene delivery to the retina. Genes 2019;10. doi:10.3390/genes10040287. [Epub ahead of print: 09 Feb 2019].

35 Dong JY, Fan PD, Frizzell RA. Quantitative analysis of the packaging capacity of recombinant adeno-associated virus. Hum Gene Ther 1996:7:2101-12.

36 Trapani I, Colella P, Sommella A, et al. Effective delivery of large genes to the retina by dual AAV vectors. EMBO Mol Med 2014:6:194-211.

37 Arbabi A, Liu A, Ameri H. Gene therapy for inherited retinal degeneration. J Ocul Pharmacol Ther 2019:35:79-97.

38 Allocca M, Mussolino C, Garcia-Hoyos M, et al. Novel adeno-associated virus serotypes efficiently transduce murine photoreceptors. J Virol 2007;81:11372-80.

39 Petrs-Silva H, Dinculescu A, Li Q, et al. Novel properties of tyrosine-mutant AAV2 vectors in the mouse retina. Mol Ther 2011;19:293-301.

40 Mingozzi F, High KA. Immune responses to AAV in clinical trials. Curr Gene Ther 2011;11:321-30.
41 Zaiss AK, Muruve DA. Immune responses to adeno-associated virus vectors. Curr Gene Ther 2005:5:323-31.

42 Li Q, Miller R, Han P-Y, et al. Intraocular route of AAV2 vector administration defines humoral immune response and therapeutic potential. Mol Vis 2008:14:1760-9.

43 Planul A, Dalkara D. Vectors and gene delivery to the retina. Annu Rev Vis SCi 2017;3:121-40.

44 MacLaren RE, Groppe M, Barnard AR, et al. Retinal gene therapy in patients with choroideremia: initial findings from a phase 1/2 clinical trial. Lancet 2014;383:1129-37.

45 Bruewer AR, Mowat FM, Bartoe JT, et al. Evaluation of lateral spread of transgene expression following subretinal AAV-mediated gene delivery in dogs. PLoS One 2013;8:e60218.

46 Stieger K, Colle M-A, Dubreil L, et al. Subretinal delivery of recombinant AAV serotype 8 vector in dogs results in gene transfer to neurons in the brain. $\mathrm{Mol}$ Ther 2008;16:916-23.

47 Xue K, Groppe M, Salvetti AP, et al. Technique of retinal gene therapy: delivery of viral vector into the subretinal space. Eye 2017:31:1308-16

48 Dalkara D, Kolstad KD, Caporale N, et al. Inner limiting membrane barriers to AAVmediated retinal transduction from the vitreous. Mol Ther 2009:17:2096-102.

49 Reid CA, Ertel KJ, Lipinski DM. Improvement of photoreceptor targeting via intravitrea delivery in mouse and human retina using combinatory rAAV2 capsid mutant vectors. Invest Ophthalmol Vis Sci 2017:58:6429-39.

50 Yin L, Greenberg K, Hunter JJ, et al. Intravitreal injection of AAV2 transduces macaque inner retina. Invest Ophthalmol Vis Sci 2011:52:2775-83.

51 Dalkara D, Byrne LC, Klimczak RR, et al. In vivo-directed evolution of a new adenoassociated virus for therapeutic outer retinal gene delivery from the vitreous. Sci Trans Med 2013; 5:189ra76

52 Jüttner J, Szabo A, Gross-Scherf B, et al. Targeting neuronal and glial cell types with synthetic promoter AAVs in mice, non-human primates and humans. Nat Neurosci 2019;22:1345-56

53 Ali RR, Reichel MB, De Alwis M, et al. Adeno-Associated virus gene transfer to mouse retina. Hum Gene Ther 1998;9:81-6.

54 Hellström M, Ruitenberg MJ, Pollett MA, et al. Cellular tropism and transduction properties of seven adeno-associated viral vector serotypes in adult retina after intravitreal injection. Gene Ther 2009:16:521-32.

55 Habot-Wilner Z, Noronha G, Wykoff CC. Suprachoroidally injected pharmacological agents for the treatment of chorio-retinal diseases: a targeted approach. Acta Ophthalmol 2019;97:460-72.

56 Ding K, Shen J, Hafiz Z, et al. AAV8-vectored suprachoroidal gene transfer produces widespread ocular transgene expression. J Clin Invest 2019;130:4901-11.

57 Peden MC, Min J, Meyers C, et al. Ab-Externo AAV-mediated gene delivery to the suprachoroidal space using a 250 micron flexible microcatheter. PLoS One 2011:6:e17140.

58 Lewis RA, von Wolff $\mathrm{K}$, Tetz M, et al. Canaloplasty: circumferential viscodilation and tensioning of Schlemm's canal using a flexible microcatheter for the treatment of open-angle glaucoma in adults: interim clinical study analysis. J Cataract Refract Surg 2007:33:1217-26.

59 Jung JH, Chae JJ, Prausnitz MR. Targeting drug delivery within the suprachoroidal space. Drug Discov Today 2019:24:1654-9.

60 Tetz M, Rizzo S, Augustin AJ. Safety of submacular suprachoroidal drug administration via a microcatheter: retrospective analysis of European treatment results. Ophthalmologica 2012;227:183-9.

61 Yeh S, Kurup SK, Wang RC, et al. Suprachoroidal injection of triamcinolone acetonide, CLS-TA, for macular edema due to noninfectious uveitis: a randomized, phase 2 study (DOGWOOD). Retina 2019;39:1880-8.

62 Rasmussen H, Chu KW, Campochiaro P, et al. Clinical protocol. An open-label, phase I, single administration, dose-escalation study of ADGVPEDF.11D (ADPEDF) in neovascular age-related macular degeneration (AMD). Hum Gene Ther 2001;12:2029-32.

63 Campochiaro PA, Nguyen QD, Shah SM, et al. Adenoviral vector-delivered pigment epithelium-derived factor for neovascular age-related macular degeneration: results of a phase I clinical trial. Hum Gene Ther 2006;17:167-76.

64 Kendall RL, Thomas KA. Inhibition of vascular endothelial cell growth factor activity by an endogenously encoded soluble receptor. Proc Natl Acad Sci U SA 1993:90:10705-9.

65 Rakoczy EP, Lai C-M, Magno AL, et al. Gene therapy with recombinant adenoassociated vectors for neovascular age-related macular degeneration: 1 year followup of a phase 1 randomised clinical trial. Lancet 2015;386:2395-403.

66 Constable IJ, Pierce CM, Lai C-M, et al. Phase 2A randomized clinical trial: safety and post hoc analysis of subretinal rAAV.sFLT-1 for wet age-related macular degeneration. EBioMedicine 2016:14:168-75.

67 Heier JS, Kherani S, Desai S, et al. Intravitreous injection of AAV2-sFLT01 in patients with advanced neovascular age-related macular degeneration: a phase 1, open-label trial. Lancet 2017:390:50-61.

68 Grishanin R, Vuillemenot B, Sharma P, et al. Preclinical evaluation of ADVM-022, a novel gene therapy approach to treating wet age-related macular degeneration. $\mathrm{Mol}$ Ther 2019;27:118-29. 
69 Kiss S. 24-Week cohort 1 data from the optic trial - intravitreal gene therapy with ADVM-022 (AAV.7m8-aflibercept) for neovascular age-related macular degeneration. London: Retina Society Annual Meeting, 2019.

70 Kiss S. Interim 24-week cohort 1 data from the optic trial -Intravitreal gene therapy with ADVM-022 (AAV.7m8-aflibercept) for neovascular age-related macular degeneration. San Francisco, 2019.

71 Wykoff CC. Phase 1 study of intravitreal gene therapy with ADVM-022 for neovascular age-related macular degeneration (optic trial cohort 1). New York: ACRC Macula, 2020. http://investors.adverum.com/static-files/f233ae69-89e3-4c69-85719c12e33de011

72 REGENXBIO. REGENXBIO reports continued progress across programs in Year-End 2019 corporate update, 2020. Available: https://www.prnewswire.com/news-releases/ regenxbio-reports-continued-progress-across-programs-in-year-end-2019-corporateupdate-300983973.html [Accessed 21 Jan 2020].

73 REGENXBIO. REGENXBIO Announces additional positive interim phase I/lla trial update for RGX-134 for the treatment of wet AMD at the American Academy of ophthalmology 2019 annual meeting, 2019. Available: https://www.prnewswire. com/news-releases/regenxbio-announces-additional-positive-interim-phase-iiiatrial-update-for-rgx-314-for-the-treatment-of-wet-amd-at-the-american-academyof-ophthalmology-2019-annual-meeting-300937385.html [Accessed 10 Dec 2019].

74 AA0 2019 Daily. Gene therapy for wet AMD? 2019. Available: https://www.aao.org/ eyenet/academy-live/detail/gene-therapy-wet-amd-3 [Accessed 10 Dec 2019].

75 Binley K, Widdowson PS, Kelleher M, et al. Safety and biodistribution of an equine infectious anemia virus-based gene therapy, RetinoStat $(\Theta)$, for age-related macular degeneration. Hum Gene Ther 2012;23:980-91.

76 Campochiaro PA, Lauer AK, Sohn EH, et al. Lentiviral vector gene transfer of Endostatin/Angiostatin for macular degeneration (GEM) study. Hum Gene Ther 2017;28:99-111.
77 Kumar-Singh R. The role of complement membrane attack complex in dry and wet AMD - From hypothesis to clinical trials. Exp Eye Res 2019;184:266-77.

78 Cashman SM, Ramo K, Kumar-Singh R. A non membrane-targeted human soluble CD59 attenuates choroidal neovascularization in a model of age related macular degeneration. PLOS One 2011;6:e19078.

79 Andrae J, Gallini R, Betsholtz C. Role of platelet-derived growth factors in physiology and medicine. Genes Dev 2008;22:1276-312.

80 Hussain RM, Ciulla TA. Emerging vascular endothelial growth factor antagonists to treat neovascular age-related macular degeneration. Expert Opin Emerg Drugs 2017;22:235-46.

81 Augustin HG, Koh GY, Thurston G, et al. Control of vascular morphogenesis and homeostasis through the angiopoietin-Tie system. Nat Rev Mol Cell Biol 2009;10:165-77.

82 Lee J, Park D-Y, Park DY, et al. Angiopoietin-1 suppresses choroidal neovascularization and vascular leakage. Invest Ophthalmol Vis Sci 2014;55:2191-9.

$83 \mathrm{Kim} \mathrm{J}$, Park JR, Choi J, et al. Tie2 activation promotes choriocapillary regeneration for alleviating neovascular age-related macular degeneration. Sci Adv 2019;5:eaau6732.

84 Xia X-bo, Xiong S-qi, Song W-tao, et al. Inhibition of retinal neovascularization by siRNA targeting VEGF(165). Mol Vis 2008;14:1965-73.

85 Garba AO, Mousa SA. Bevasiranib for the treatment of wet, age-related macular degeneration. Ophthalmol Eye Dis 2010;2:OED.S4878-83.

86 Kaiser PK, Symons RCA, Shah SM, et al. Rnai-Based treatment for neovascular agerelated macular degeneration by Sirna-027. Am J Ophthalmol 2010;150:33-9.

87 Askou AL, Pournaras J-AC, Pihlmann M, et al. Reduction of choroidal neovascularization in mice by adeno-associated virus-delivered anti-vascular endothelial growth factor short hairpin RNA. J Gene Med 2012;14:632-41.

88 Pihlmann M, Askou AL, Aagaard L, et al. Adeno-Associated virus-delivered polycistronic microRNA-clusters for knockdown of vascular endothelial growth factor in vivo. J Gene Med 2012;14:328-38. 